\title{
Translocation of indigenous microflora in an experimental model of sepsis
}

\author{
P. NAABER, I. SMIDT, K. TAMME*, A. LIIGANT†, H. TAPFER $\dagger$, M. MIKELSAAR and R. TALVIK* \\ Department of Microbiology, *Department of Anaesthesiology and Intensive Care and †Department of \\ Anatomy, University of Tartu, Tartu, Estonia
}

\begin{abstract}
Translocation of viable bacteria from gut to bloodstream and other sterile body sites during shock has been demonstrated in several experimental and clinical studies. The factors causing translocation and its incidence at different stages of shock are not known. The aim of the study was to evaluate the importance of several factors causing translocation of indigenous microflora in an experimental model of septic shock based on intraperitoneal Escherichia coli sepsis in rats. Counts of inoculated $E$. coli and translocated bacteria in different locations, gut morphology and haematological values were evaluated at different stages of sepsis. Sepsis developed in all animals and $E$. coli achieved the highest counts in blood $6 \mathrm{~h}$ after inoculation. Translocation was commonest at 6 and $12 \mathrm{~h}$ after inoculation. Frequently translocating bacteria were lactobacilli, bifidobacteria, bacteroides and peptostreptococci. In early sepsis, translocation was associated with high $E$. coli counts in blood, yet in late sepsis the opposite correlation was present. Low infiltration by neutrophils in the ileum and decreased mitotic activity in the colon were associated with a high translocation rate. In early sepsis, translocation was associated with low lymphocyte counts, but in late sepsis, with low neutrophil counts. Translocation of bacteria (including anaerobes) that colonise the gut in high counts takes place during sepsis. Putative influencing factors such as activity of the primary disease (bacterial counts in blood), gut morphology or haematological values seem to have different impacts on translocation, depending on the stage of the disease.
\end{abstract}

\section{Introduction}

Bacterial translocation is the passage of viable indigenous bacteria to sterile body sites, such as the mesenteric lymph nodes (MLN), spleen, liver and bloodstream [1]. Although bacterial translocation can take place through several mucosal membranes, most interest has focused on the intestinal tract as potentially the most important source of translocating bacteria [14]. It is generally agreed that gram-negative facultative bacteria translocate more easily than anaerobes and gram-positive bacteria [5].

Several factors - such as bacterial overgrowth, alteration of mucosal barriers and immunosuppression promote bacterial translocation from the intestinal tract [6]. An imbalance of intestinal microflora and overgrowth by gram-negative facultative bacteria through administration of antibiotics enhances translocation

Received 20 July 1999; accepted 30 Sept. 1999.

Corresponding author: Dr P. Naaber (e-mail: paul.naaber@ kliinikum.ee).
$[1,7]$. Damage of the mucosal barrier by several factors, including haemorrhagic- and endotoxin-induced shock, can also induce bacterial translocation $[8,9]$. However, evidence for the correlation of translocation with intestinal mucosal permeability to non-bacterial compounds is still contradictory $[10,11]$.

Despite many experimental and clinical studies, it is not known whether indigenous bacteria translocate with equal efficiency from all sites of the gastrointestinal tract or whether they translocate more efficiently from certain regions [1]. Most studies investigating a possible relationship between histological changes in mucosae and translocation have focused on changes in the small bowel and only a few studies have analysed and demonstrated alterations in colonic mucosa $[12,13]$. Passage of bacteria through mucosal membranes and survival in extra-intestinal sites depend on the effectiveness of the host immune system. Both humoral and cellular immunity have been shown to be important in protection against translocation $[1,2,14]$. However, it is not known if one of them prevails at different stages of bacterial translocation. 
Thus, several physiological and pathological conditions could be associated with enhanced translocation of indigenous bacteria through mucous membranes. Although the presence of bacterial translocation during shock and other serious diseases has been demonstrated clearly in several experimental and clinical studies, the real incidence of translocation in different stages of disease, the factors to which it is related, and the influence of translocation on patient outcome are still unclear $[4,8,15,16]$.

The aim of the study was to detect the translocation of indigenous bacteria during early and late Escherichia coli sepsis in an experimental animal model and to correlate it with several sepsis-associated parameters such as E. coli counts in blood, histological changes in intestinal mucosa and haematological values.

\section{Materials and methods}

\section{Experimental animals}

Wistar rats $(\mathrm{n}=51)$ were challenged with an $E$. coli isolate from the blood of a septic patient. Viable $E$. coli cells $\left(2 \times 10^{7} / \mathrm{g}\right.$ of body weight $)$ were suspended in $1.5 \mathrm{ml}$ of saline and inoculated intraperitoneally together with $1.5 \mathrm{ml}$ of haemolysed blood. Blood was added to suppress an initial immune response in the peritoneal cavity [17]. Fourteen rats died before the end of the study and were excluded from the analysis. A total of 37 rats (median weight $241 \mathrm{~g}$ ) was included in the study and some were killed $45 \mathrm{~min}$ (group I), $2 \mathrm{~h}$ (group II), $6 \mathrm{~h}$ (group III), $24 \mathrm{~h}$ (group IV), $48 \mathrm{~h}$ (group V) or 5 days (group VI) after inoculation (Table 1). A further seven uninoculated rats served as controls. The rats had free access to standard laboratory chow and water ad libitum during the study, which was approved by the local ethical committee of animal research.

\section{Processing of bacteriological samples}

During autopsy, heart blood and samples from the mesenteric lymph nodes, liver, spleen, lung, heart muscle and kidney were taken. The organs were weighed, homogenised in phosphate buffer, serially diluted in an anaerobic chamber (Sheldon Manufacturing) and seeded on: Wilkins Chalgren Blood Agar (Oxoid, CM619) for isolation of anaerobes; Blood Agar
(Oxoid, CM271) for isolation of aerobes; Wilkins Chalgren Agar with vancomycin and nalidixic acid (Oxoid, CM619, SR108) for selective isolation of gram-negative anaerobes and Wilkins Chalgren Agar with colistin and nalidixic acid (Oxoid, CM619, SR70) for selective isolation of gram-positive organisms. Blood (125 $\mu \mathrm{l})$ was inoculated into broth (Signal Blood Culture System, Oxoid) and another $125 \mu \mathrm{l}$ were seeded on Wilkins Chalgrin blood agar. For quantitation of bacteria, serial dilutions of blood and peritoneal fluid were seeded from the above-mentioned selective and non-selective media. Blood agar plates were incubated aerobically for $48 \mathrm{~h}$ at $37^{\circ} \mathrm{C}$. Plates of the other media were incubated at $37^{\circ} \mathrm{C}$ in an anaerobic chamber for 5 days. After incubation, the colonies were counted and colonies of different morphotypes were isolated for identification to the genus level. Bacterial counts $/ \mathrm{g}$ or $/ \mathrm{ml}$ and the number of translocation cases (i.e., the sum of different organ/microbe combinations) were calculated.

\section{Processing of histological samples}

Samples from the central part of the ileum and the colon ( $3 \mathrm{~cm}$ from anus) were taken and fixed with neutral formalin $10 \%$. Paraffin sections were stained with haematoxylin and eosin, acridine orange and a modified Gram's stain. Two experienced histopathologists examined coded slides blind. Several inflammatory and destructive changes such as: hyperaemia, haemorrhages, vacuolisation, necroses, goblet cell counts, mitotic activity, diffuse lymphatic infiltration, infiltration by polymorphonuclear leucocytes (PMNLs) and the presence of microbes within crypts were evaluated. A numerical score was given to the changes: $0=$ norm; $-1,-2,-3=$ diminishing (mild, moderate, severe changes) as compared with the norm; 1, 2, $3=$ increasing changes as compared with the norm. The norm was defined by the examination of control rats as in a previous study [18].

\section{Counting of blood cells}

Blood samples from all killed animals were collected into standard EDTA-coated test tubes. Total counts of white blood cells (WBC) and lymphocytes were measured in a Sysmex K 1000 machine (TOA Medical Electronics, Kobe, Japan). Blood smears were made

Table 1. The groups of animals and the samples collected

\begin{tabular}{lll}
\hline Group & Time of killing & Samples \\
\hline Control & 2 days & PC, HB, MLN, Li, Sp, Lu, HM, Ki, Co, Il \\
I & $45 \mathrm{~min}$ & PC, HB, MLN, Li, Sp, Co, Il \\
II & $2 \mathrm{~h}$ & PC, HB, MLN, Li, Co, Il \\
III & $6 \mathrm{~h}$ & PC, HB, MLN, Li, Co, Il \\
IV & $24 \mathrm{~h}$ & PC, HB, MLN, Li, Co, Il \\
V & $48 \mathrm{~h}$ & PC, HB, MLN, Li, Sp, Lu, HM, Ki, Co, Il \\
VI & 5 days & PC, HB, MLN, Li, Sp, Lu, HM, Ki, Co, Il \\
\hline
\end{tabular}

Bacteriological studies: PC, peritoneal cavity; HB, heart blood; MLN, mesenteric lymph nodes; $\mathrm{Li}$, liver; Sp, spleen; Lu, lung; HM, heart muscle; Ki, kidney. Histological studies: Co, colon; Il, ileum. 
and stained according to Pappenheim for counting neutrophils, monocytes and eosinophils.

\section{Statistical analysis}

Data were analysed by a Jandel SigmaStat 2.0 program. The following tests were used: unpaired $t$ test for comparison of groups with normally distributed data, Mann-Whitney rank sum test for comparison of groups without normal distribution, Spearman rank order correlation to quantify the relationship between several variables and the $\chi^{2}$ or Fisher test for prevalence comparison of changes between groups.

\section{Results}

\section{Development of sepsis}

The model of infection was suitable for investigation of different stages of sepsis. All inoculated animals survived at least $6 \mathrm{~h}$; during the following days a lethal infection developed in some animals, but others survived. The survival rate of the animals over 5 days was: $100 \%$ at $6 \mathrm{~h} ; 85 \%$ at 1 day; $77 \%$ at 2 days and $62 \%$ at 5 days. All control animals remained asymptomatic. Because different trends were found in several parameters of animals killed at different times after inoculation, the animals were grouped into those with early sepsis $(45 \mathrm{~min}-24 \mathrm{~h}$ ) and those with late sepsis (2-5 days).

The dissemination of $E$. coli from the peritoneal cavity to sterile sites was detected as early as $45 \mathrm{~min}$ after inoculation. The counts of $E$. coli were highest in the blood of animals in group III ( $6 \mathrm{~h}$ after inoculation) (Fig. 1). Significantly lower counts in blood were found in rats from groups I, II, V and VI. Similar trends were seen in the $E$. coli counts in the mesenteric lymph nodes, liver and peritoneal cavity. When comparisons were made between the counts of E. coli in blood and other organs of animals in the same group, it was found that the counts in the peritoneal cavity remained higher than those in blood during early sepsis (median 8.1 versus $5.6 ; \mathrm{p}<0.001$ ) and late sepsis (median 7.8 versus 5.6; $\mathrm{p}<0.001)$. In other organs, the counts of $E$. coli did not differ significantly from those in blood. When the counts in individual animals were correlated, a strong positive association was found between the counts of E. coli in blood and in other organs $(\mathrm{p}<0.001)$. Correlation coefficients $(r)$ were: mesenteric lymph nodes, $r=0.79$; liver, $r=0.85$; peritoneal cavity, $r=0.83$, spleen, $r=0.83$; lungs, $r=0.88$; kidney, $r=0.9$; heart muscle, $r=0.84$.

\section{Translocation of normal microflora to sterile body sites}

No cases of translocation were present in the control group. In the test group only a few cases of translocation were detected $45 \mathrm{~min}$ after inoculation (group I animals) (Table 2). In group VI animals (5 days after inoculation) translocation was also relatively rare. Bacterial translocation was most frequent in groups II-V, i.e., in animals $2-48 \mathrm{~h}$ after inoculation with E. coli. In early sepsis, the highest incidence of translocation was seen in the mesenteric lymph nodes. In late sepsis, bacteria were found most frequently in kidney and lungs. Indigenous bacteria were recovered from blood in only a few cases. The most commonly translocating bacteria were lactobacilli, bifidobacteria, bacteroides and peptostreptococci which were found together in $63 \%$ of all cases of translocation (Fig. 2). The least frequently translocated bacteria were enterococci, staphylococci, streptococci, propionibacteria, eubacteria and gram-negative non-fermenters. If translocation was present, one or two bacterial groups were usually recovered from the same organ in early sepsis. In late sepsis, translocation varied more between individual animals: in some rats no translocation to any organs was detected, but in others up to seven

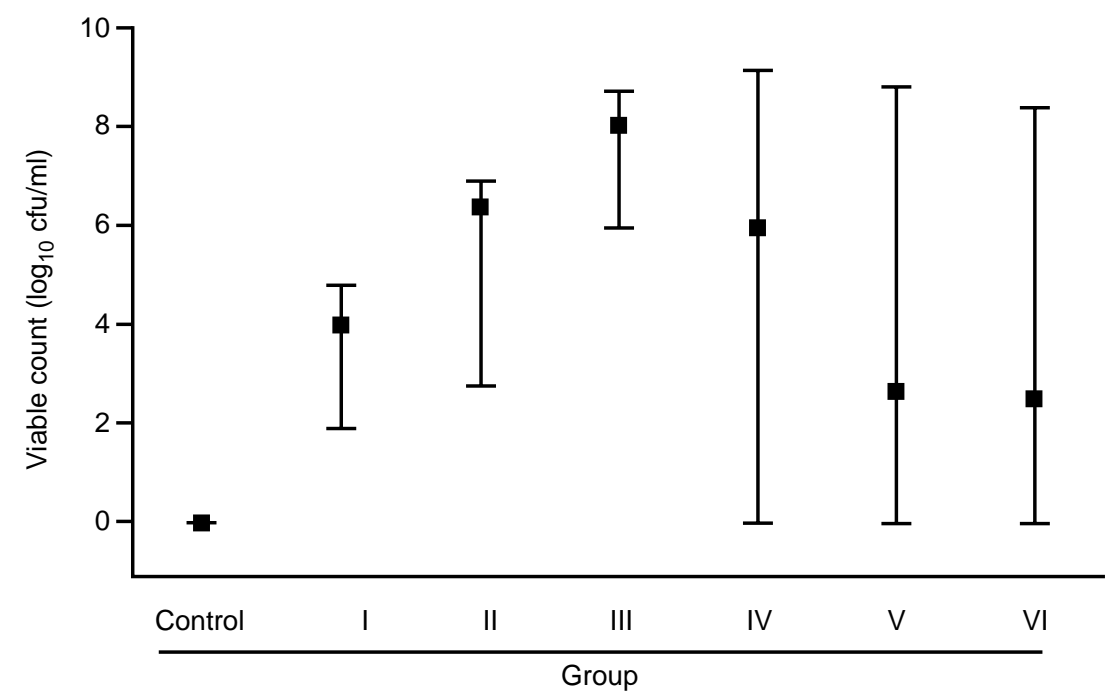

Fig. 1. The counts of E. coli in heart blood (range | and median $\mathbf{\square})$. Statistically significant differences: group I versus II $(\mathrm{p}<0.05)$; group I versus III $(\mathrm{p}<0.001)$; group III versus $\mathrm{V}(\mathrm{p}<0.05)$; group III versus VI $(\mathrm{p}<0.05)$. 
Table 2. The frequency of bacterial translocation and the organs involved

\begin{tabular}{lccccc}
\hline & \multicolumn{3}{c}{ Number of animals with/without translocation } \\
\cline { 2 - 5 } Organ & Group I & Group II & Group III & Group IV & Group V \\
\hline Blood & $0 / 6$ & $1 / 5$ & $1 / 5$ & $0 / 6$ & $1 / 4$ \\
MLN & $2 / 4$ & $6 / 0$ & $6 / 0$ & $5 / 1$ & $4 / 1$ \\
Liver & $1 / 5$ & $4 / 2$ & $5 / 1$ & $4 / 2$ & $3 / 2$ \\
Spleen & $0 / 6$ & ND & ND & ND & $1 / 4$ \\
Lung & ND & ND & ND & ND & $4 / 1$ \\
Heart muscle & ND & ND & ND & ND & $3 / 2$ \\
Kidney & ND & ND & ND & ND & $4 / 8$ \\
\hline
\end{tabular}

$\mathrm{ND}$, not determined. No cases of translocation were found in the control group. Significant differences between groups: MLN, II versus VI $(\mathrm{p}=0.01)$, III versus VI $(\mathrm{p}=0.01)$; liver, I versus III $(\mathrm{p}<0.05)$, VI versus II $(\mathrm{p}<0.05)$, VI versus III ( $p<0.01)$, VI versus IV ( $<<0.05)$, VI versus $\mathrm{V}(\mathrm{p}<0.05)$.

different bacterial groups were isolated from the same organ. The counts of indigenous bacteria from normally sterile organs were up to $\log _{10} 4.6 \mathrm{cfu} / \mathrm{g}$.

\section{Histological studies of intestinal mucosa}

There were no histological changes in the mucosal samples of the control animals except for a mild reduction in mitotic activity in one rat. At $45 \mathrm{~min}$ after inoculation (group I animals), only mild changes (reduction of mitotic activity and infiltration by lymphocytes and PMNLs) were seen in a few rats. In the group II rats ( $2 \mathrm{~h}$ after inoculation) deviations from the norm were more pronounced. In most of these animals hyperaemia, vacuolisation, reduction of goblet cells, decreased mitotic activity and infiltration by lymphocytes and PMNLs were detected. However, the expression of these characteristics in individual animals within the groups was highly variable. Because of this, no statistical difference between groups II-VI (as well as early and late sepsis) could be demonstrated. In all group II-VI rats, different morphotypes of bacteria were found within the base of the crypts of the ileum (Fig. 3). In control and most group II animals, bacteria were not detected in the crypts.

In comparisons of particular changes in the ileum and colon of individual animals a higher reduction of goblet cells $(p<0.05)$ was found and a more pronounced lymphatic infiltration $(p<0.005)$ in the ileum. No significant differences were detected between the ileal and colonic mucosa in the prevalence or intensity of other characteristics.

Correlation between the counts of E. coli, translocation of indigenous microflora and histological changes in mucosae

Correlation between the counts of E. coli and histological changes in mucosae. In early sepsis, E. coli counts in blood correlated positively with several particular and total mucosal changes in the ileum (Table 3). Correlation with changes in the colon was weaker. In late sepsis, the association between E. coli counts in blood and changes in the ileum was weak and not statistically significant. In the case of the colon, the association with most histological characters was weakly negative, but the total score of changes in the colon gave a significant negative correlation.

Correlation between the counts of E. coli and translocation of indigenous bacteria. In early sepsis, the counts of $E$. coli in blood were positively associated with total counts of translocated bacteria to primarily sterile organs (Fig. 4a) as well as the number of translocation cases $(r=0.66, \mathrm{p}<0.001)$. In late sepsis the opposite trends were found. There was a negative correlation between the counts of $E$. coli in blood and the total counts of translocated bacteria (Fig. 4b).

Correlation between translocation of indigenous bacteria and histological changes in mucosae. In early sepsis, the number of translocation cases was negatively associated with mitotic activity in the colon $(r=-0.71, \mathrm{p}=0.001)$ and positively with the presence of microbes in the crypts of the colon $(r=0.52$, $\mathrm{p}<0.05)$ and a total score of histological changes in the colon $(r=0.52, \mathrm{p}<0.05)$. The total counts of translocated bacteria were in negative correlation with mitotic activity in the colon $(r=-0.5, \mathrm{p}<0.05)$.

In late sepsis a significant negative association was found only between infiltration of PMNLs in the ileum and the number of translocation cases and counts of bacteria translocated into blood, MLN and liver (in both cases $r=-0.67, \mathrm{p}<0.05)$.

Correlating translocation and the histology of mucosae of all infected animals, it was found that higher incidences of translocation cases were significantly associated with decreased mitotic activity in the colon $(r=-0.5, \mathrm{p}<0.01)$ and lower infiltration by PMNLs in the ileum $(r=-0.62, \mathrm{p}<0.05)$.

Correlation of E. coli counts and translocation with haematological values. A significant negative correlation was found between the counts of $E$. coli in blood and the total count of white blood cells $(r=-0.96$, $\mathrm{p}<0.001)$. This association was present in early $(r=-0.64, \quad \mathrm{p}<0.005) \quad$ as well as late sepsis $(r=-0.79, \quad \mathrm{p}<0.05)$. A similar association was 


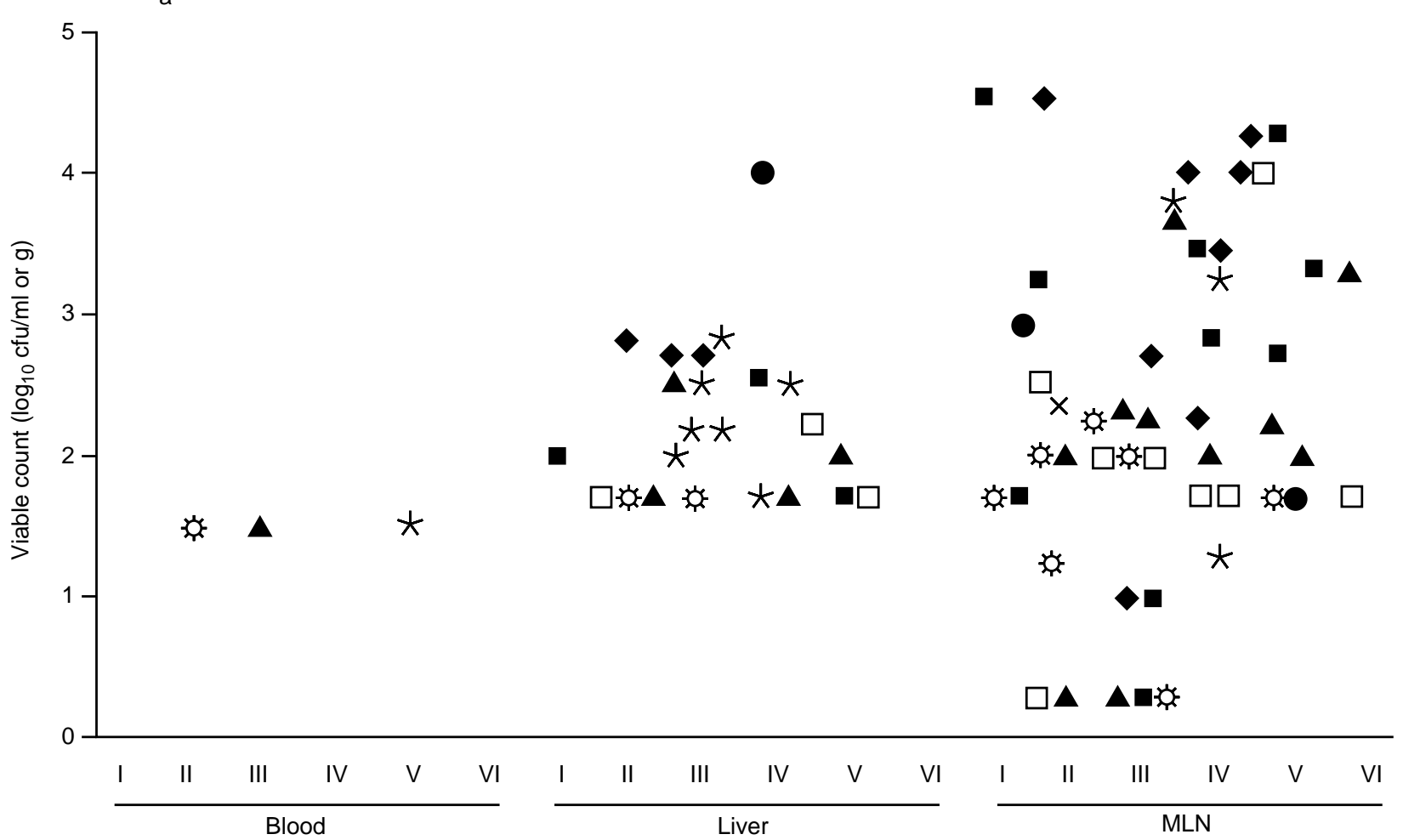

b

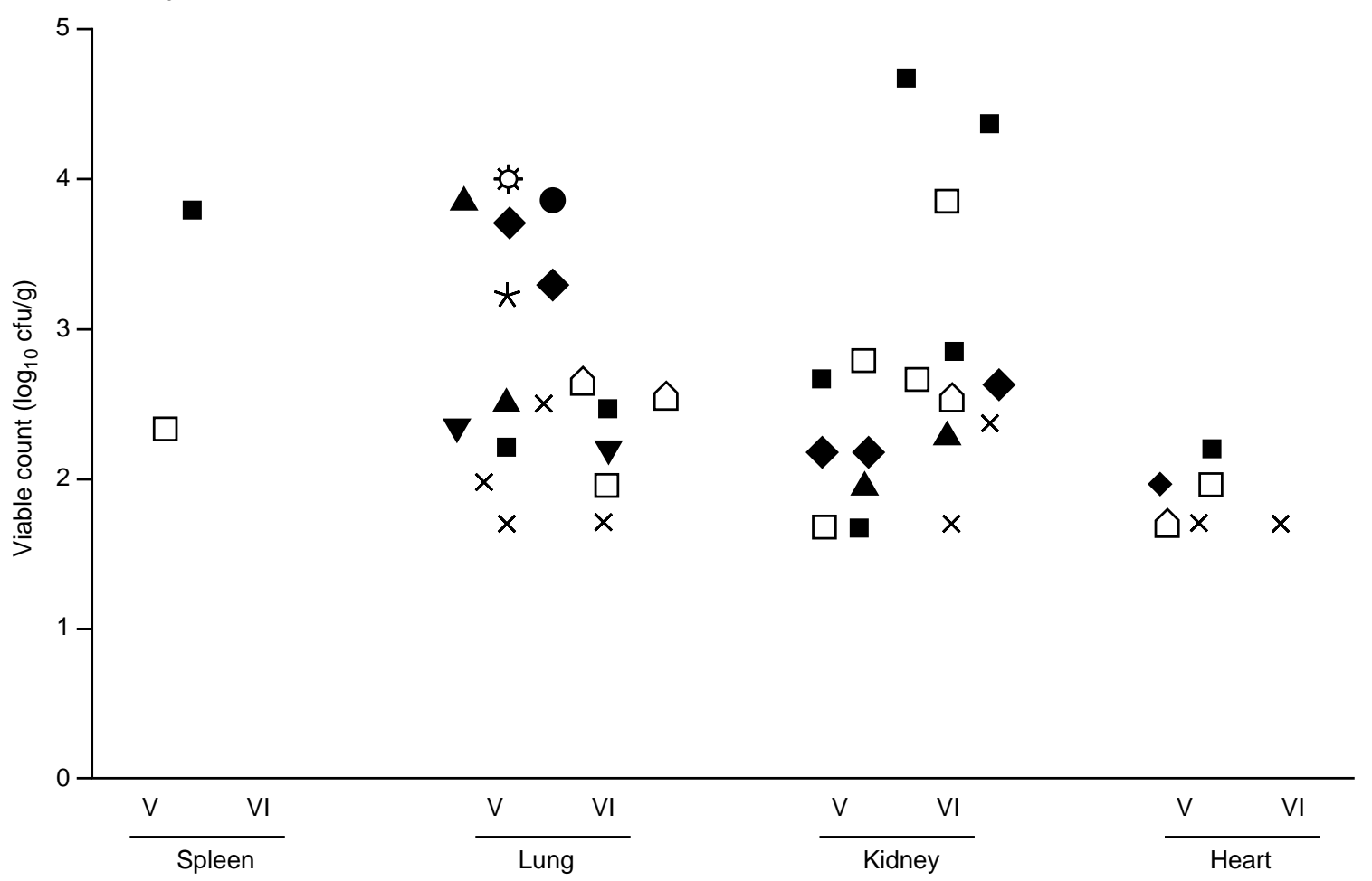

Fig. 2. (a) Bacterial translocation of indigenous microflora to blood, liver and mesenteric lymph nodes (MLN) in rats of groups I-VI. Lactobacilli $\mathbf{\square}$; bifidobacteria $\square$; bacteroides $\boldsymbol{\Delta}$; peptostreptococci $\bullet$; coliforms $\star$; enterococci staphylococci $\times$; streptococci - . (b) Bacterial translocation of indigenous microflora to spleen, lung, kidney and heart in rats of groups V and VI. Lactobacilli $\mathbf{\square}$; bifidobacteria $\square$; bacteroides $\boldsymbol{\Delta}$; peptostreptococci $\bullet$; coliforms $\star$; enterococci ; staphylococci $\times$; streptococci 0 ; glucose non-fermenting gram-negative organisms $\vec{\nabla}$; propionibacteria $\triangle$ 


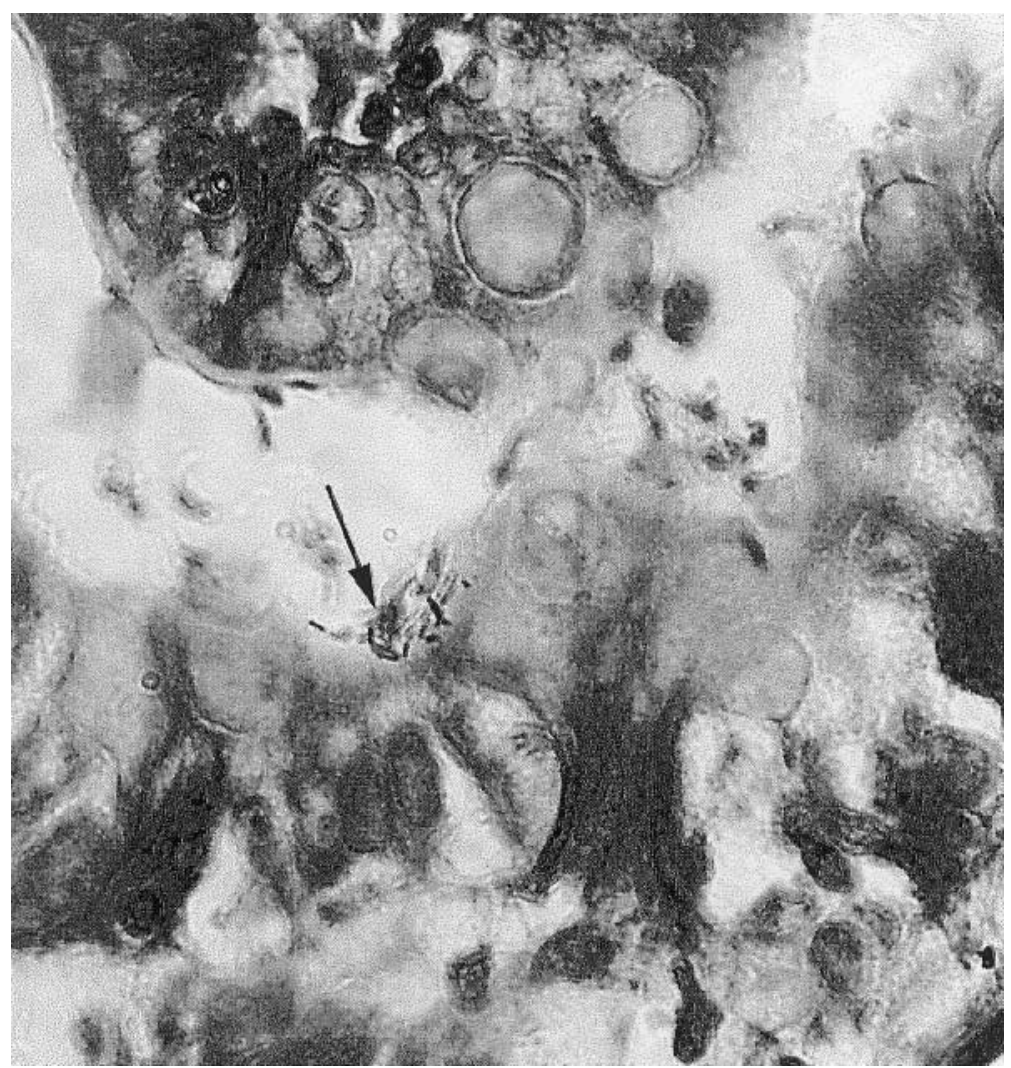

Fig. 3. Different morphotypes of bacteria (arrow) found in the connective tissue at the base of the crypts of ileum (modified Gram's stain $\times 900$ ).

Table 3. Significant correlations between E. coli counts and histological changes in mucosae

\begin{tabular}{lccc}
\hline & \multicolumn{3}{c}{ Correlation coefficient $/ \mathrm{p}$} \\
\cline { 2 - 4 } Histological changes & Early sepsis & Late sepsis & All \\
\hline Hyperaemia in ileum & $0.47 /<0.05$ & $\mathrm{NS}$ & $\mathrm{NS}$ \\
Haemorrhage in ileum & $0.53 /<0.05$ & $\mathrm{NS}$ & $\mathrm{NS}$ \\
Necrosis in ileum & $0.54 /<0.01$ & $\mathrm{NS}$ & $\mathrm{NS}$ \\
Microbes in crypts of ileum & $0.47 /<0.05$ & $\mathrm{NS}$ & $\mathrm{NS}$ \\
Microbes in crypts of colon & $0.62 /<0.01$ & $\mathrm{NS}$ & $\mathrm{NS}$ \\
Infiltration with PMNLs in ileum & $\mathrm{NS}$ & $\mathrm{NS}$ & $0.43 /<0.05$ \\
Total score of ileum & $0.68 /<0.01$ & $\mathrm{NS}$ & $0.44 /<0.05$ \\
Total score of colon & $\mathrm{NS}$ & $-0.6 /<0.05$ & $\mathrm{NS}$ \\
Total score of ileum and colon & $0.60 /<0.05$ & $\mathrm{NS}$ & $\mathrm{NS}$ \\
\hline
\end{tabular}

NS, not significant.

present between the counts of E. coli and lymphocytes in blood $(r=-0.79, \mathrm{p}<0.001)$.

Correlating haematological values with translocation, it was found that in early sepsis the number of translocation cases was negatively associated with the counts of lymphocytes in blood $(r=-0.7, \mathrm{p}<0.05)$, but in late sepsis with the counts of neutrophils $(r=-0.65, \mathrm{p}<0.01)$.

\section{Discussion}

Many different experimental models have been developed to determine the rate and importance of bacterial translocation. In most models, translocation has been detected at one or two fixed times after a single challenge or alteration, e.g., injection of endotoxin. In the rat model used in the present study, the continuous liberation of endotoxin and modulation of the immune response take place during development of sepsis. Translocation to several organs and histological changes in different intestinal sites were determined at different times during early and late sepsis. Although the interpretation of these results is probably more complicated, we believe that this model provides a better reproduction of the actual clinical development of sepsis.

E. coli translocated rapidly from the peritoneal cavity to blood and other organs within $45 \mathrm{~min}$ and achieved the highest counts after $6 \mathrm{~h}$. In the primary inoculation 


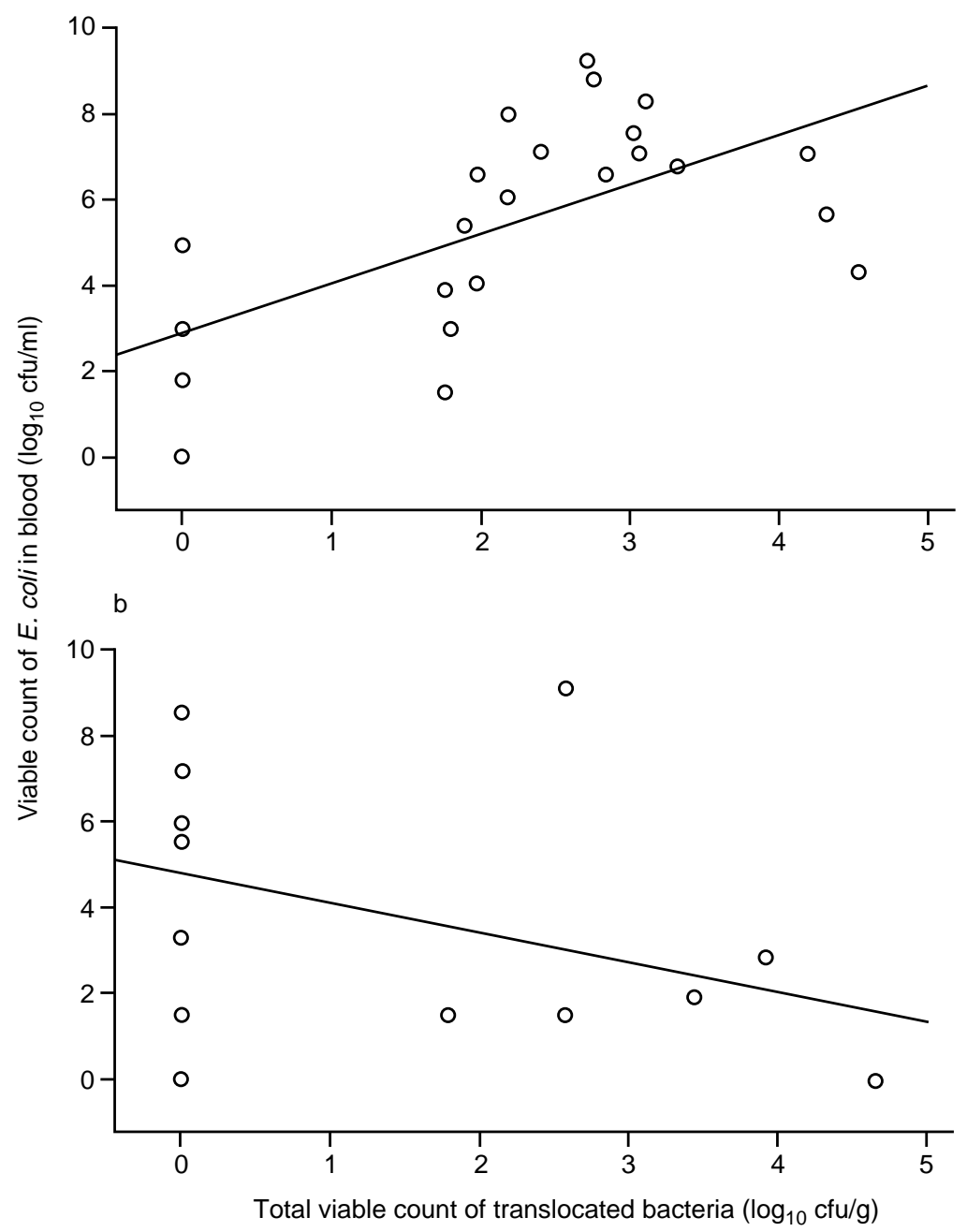

Fig. 4. (a) Correlation $(r=0.62 ; \mathrm{p}=0.001)$ between the counts of $E$. coli in blood and counts of translocated bacteria in early sepsis. (b) Correlation $(r=-0.59 ; \mathrm{p}=0.03)$ between the counts of $E$. coli in blood and counts of translocated bacteria in late sepsis.

site - the peritoneal cavity - the counts of E. coli were significantly higher than those in blood and other organs throughout the whole experiment. There was a strong positive correlation between the counts of $E$. coli in the peritoneal cavity and in blood. This indicates that the most active proliferation of bacteria takes place in the primary infection site. From this site bacteria disseminate to blood and almost equally to other organs.

High counts of E. coli were found to be strongly associated with low counts of lymphocytes and total WBC. While on the one hand, the high counts of $E$. coli and subsequent liberation of high amounts of endotoxin and inflammatory mediators lead to a more pronounced dysfunction and destruction of immune cells [19], on the other hand, higher counts of WBC and lymphocytes could serve as indicators of a more active immune response, effectively eradicating bacteria from blood.

Gram-negative sepsis led to massive bacterial translocation, mainly to MLN and the liver, that started as early as $45 \mathrm{~min}$ after inoculation and achieved the highest incidence between 2 and $24 \mathrm{~h}$ after challenge with bacteria. In early sepsis, the translocation to MLN and liver was present in most animals, whereas in late sepsis individual differences appeared. In some animals, translocation of many different bacteria to several organs was present, but in others, the translocation was absent or only few a species of bacteria were found in one or two organs. Translocating microbes were found to be mainly indigenous anaerobes or facultative bacteria such as lactobacilli, bifidobacteria, bacteroides and peptostreptococci, bacteria that normally colonise the intestine in high numbers. A mainly protective function has been attributed to these bacteria. Although it was not possible to distinguish indigenous $E$. coli from the inoculated strain, the translocation of other coliforms and aerobes was relatively rare. Most previous investigators have attributed the main role in bacterial translocation to aerobic and facultative gramnegative bacteria. Yet, as in this study, some other studies have also shown the high translocation rate of anaerobes $[2,20]$. It is possible that there are no major differences between the translocation rates of faculta- 
tive and anaerobic bacteria, but that the coliforms survive better in the bloodstream and lymph nodes than anaerobes, as some authors have speculated previously $[2,5]$. The massive liberation of endotoxin and inflammatory modulators during development of septic shock probably suppresses the immune response against translocating bacteria and, therefore, even more susceptible bacteria such as anaerobes can survive and cause further septic complications, as seen in this study. If this is true, in clinical cases of severe sepsis and shock, the concepts of selective decontamination of the gut and selection of antibiotic prophylactics and empirical therapy of septic complication during shock should be revised.

Correlating the counts of E. coli in blood and translocation (count of translocated bacteria and prevalence) showed that, in early sepsis, high E. coli counts were clearly associated with the increase of translocation of indigenous bacteria. Surprisingly, the opposite trend was present in late sepsis. One hypothesis to explain this paradox could be the probable modification of the immune response during development of sepsis. It is known that endotoxin induces liberation of different pro- and anti-inflammatory mediators. In early sepsis, increased permeability of intestinal mucosae to bacterial translocation because of direct action of endotoxin or via different mediators leads to massive translocation of bacteria prevailing on mucosae. The finding that counts of E. coli in blood correlated well with several changes in mucosae in early sepsis supports this opinion. It is possible that suppression of the immune system enables translocated bacteria to survive. It may be that in late sepsis the presence of $E$. coli in blood induced an immune response against translocating bacteria, or alternatively, translocated bacteria caused beneficial non-specific immunostimulation against $E$. coli. Moreover, at this stage no correlation between the counts of $E$. coli and particular changes in mucosae was seen and the sum of all scores of colon alterations was even negatively associated with $E$. coli counts.

Higher counts of neutrophils in blood were associated with a rarer detection of indigenous bacteria in sterile organs in late but not in early sepsis. It is known that the trapping of immune cells to microcirculation takes place in the early stages of sepsis [21]. Higher counts of neutrophils in blood in late sepsis probably indicated more active reproduction of these cells. In these animals, neutrophils have an important role in killing invading bacteria. Correlation between translocation and dysfunction of cell-mediated immunity has also been shown in other studies [22].

Some morphological abnormalities in intestinal mucosae were present in all infected animals. After $45 \mathrm{~min}$ of bacterial challenge, only mild changes in a few characteristics were seen. By $2 \mathrm{~h}$ after inoculation, moderate to severe mucosal changes were present.
However, high variability in particular characteristics between animals even within the groups was present. Reduction of mitotic activity, lymphatic infiltration and presence of bacteria within the crypts were the most common changes detected in nearly all infected animals. Previous animal experiments have shown more pronounced changes in the ileum and caecum than in the colon after administration of endotoxin for induction of shock [1]. Surprisingly, a greater reduction of goblet cells and more expressed lymphatic infiltration in the ileum were the only differences found between the changes in ileum and colon.

Correlating changes in mucosae with translocation, no clear association was found between translocation and severe destructive morphological changes in mucosae, such as necrosis and haemorrhage. The decreased mitotic activity that was found in most infected animals correlated with higher levels of translocation. This change was probably one indicator of mucosal dysfunction during endotoxin-derived shock. In early sepsis there was also correlation between translocation and the total score of mucosal changes in the colon, but not in the ileum. These findings are contrary to the opinions of some others that associate translocation with changes in ileal mucosa $[1,12]$. On the other hand, the results of the present study indicate that higher counts of PMNLs in ileal mucosa probably have some protective function against translocation. It seems that evaluation of mucosal permeability to bacterial translocation is complex and several immunological factors, including PMNL activity, should be considered beside morphological destruction of cells.

This study revealed that translocation of different indigenous bacteria, including anaerobes, takes place during sepsis. These microbes can cause further polymicrobial septic complications in different organs. Prediction of the intensity of translocation seems to be difficult. Putative factors such as activity of primary disease (bacterial counts in blood) or haematological values seem to have a controversial impact on translocation, depending on the stage of disease. These controversial results in early and late sepsis could provide one explanation for the contradictory conclusions of several clinical studies evaluating the role and prevalence of bacterial translocation in multi-organ failure and other syndromes.

\section{References}

1. Berg RD. Translocation of enteric bacteria in health and disease. Curr Stud Hematol Blood Transfus 1992; 59: 44-65.

2. O'Boyle CJ, MacFie J, Mitchell CJ, Johnstone D, Sagar PM, Sedman PC. Microbiology of bacterial translocation in humans. Gut 1998; 42: 29-35.

3. Pierro A, van Saene HKF, Donnell SC et al. Microbial translocation in neonates and infants receiving long-term parenteral nutrition. Arch Surg 1996; 131: 176-179.

4. Reynolds JV. Gut barrier function in surgical patient. Br J Surg 1996; 83: $1668-1669$.

5. Wells CL. Relationship between intestinal microecology and 
the translocation of intestinal bacteria. Antonie Van Leeuwenhoek 1990; 58: 87-93.

6. Deitch EA, Rutan R, Waymack JP. Bacterial translocation studies of mice and men. Am $J$ Gastroenterol 1998; 93: $277-278$.

7. Mikelsaar M, Türi E. Effects of antibacterial drugs and dental surgery on the translocation of digestive tract microflora. Microecol Ther 1990; 20: 93-97.

8. Kale IT, Kuzi MA, Berkem H, Berkem R, Acar N. The presence of hemorrhagic shock increases the rate of bacterial translocation in blunt abdominal trauma. J Trauma 1998; 44: $171-174$.

9. Mishima S, Xu D, Lu Q, Deitch EA. The relationship among nitric oxide production, bacterial translocation, and intestinal injury after endotoxin challenge in vivo. J Trauma 1998; 44: $175-182$.

10. MacFie J. Bacterial translocation in surgical patients. Ann $R$ Coll Surg Engl 1997; 79: 183-189.

11. Wells CL. Colonization and translocation of intestinal bacterial flora. Transplant Proc 1996; 28: 2653-2656.

12. Lemaire LCJM, van Lanschot JJB, Stoutenbeek CP, van Deventer SJH, Wells CL, Gouma DJ. Bacterial translocation in multiple organ failure: cause or epiphenomenon are still unproven. Br J Surg 1997; 84: 1340-1350.

13. Reynolds JV, O'Farrelly C, Feigherty C et al. Impaired gut barrier function in malnourished patients. Br J Surg 1996; 83: 1288-1291.

14. Stechmiller JK, Treloar D, Allen N. Gut dysfunction in critically ill patients: a review of the literature. Am J Crit Care 1997; 6: 204-209.

15. Nieuwenhuijzen GAP, Deitch EA, Goris RJA. The relationship between gut-derived bacteria and the development of multiple organ dysfunction syndrome. J Anat 1996; 189: 537-548.

16. Saadia R. Trauma and bacterial translocation. Br J Surg 1995; 82: $1243-1244$.

17. Hau T, Hoffman BS, Simmons RL. Mechanisms of the adjuvant effect of hemoglobin in experimental peritonitis. 1. In vivo inhibition of peritoneal leukocytosis. Surgery 1978; 83: $223-229$.

18. Tapfer H, Liigant A, Talvik $\mathrm{R}$ et al. Pathomorphological responses in experimental sepsis. In: Papers on anthropology VII; University of Tartu. 1997: 339-350.

19. Ayala A, Urbanich MA, Herdon CD, Chaudry IH. Is sepsisinduced apoptosis associated with macrophage dysfunction? J Trauma 1996; 40: 568-574.

20. Naaber P, Mikelsaar R-H, Salminen S, Mikelsaar M. Bacterial translocation, intestinal microflora and morphological changes of intestinal mucosa in experimental models of Clostridium difficile infection. J Med Microbiol 1998; 47: 591-598.

21. Tighe D, Moss R, Boghossian S, Heath MF, Chessmin B, Bennett ED. Multi-organ damage resulting from experimental faecal peritonitis. Clin Sci 1989; 76: 269-276.

22. Yao Y-M, Lu L-R, Yu Y et al. Influence of selective decontamination of the digestive tract on cell-mediated immune function and bacterial/endotoxin translocation in thermally injured rats. J Trauma 1997; 42: 1073-1079. 